\title{
Distributed optical fiber sensors in concrete structures: Performance of bonding adhesives and influence of spatial resolution
}

\author{
António Barrias ${ }^{1}$, Joan R. Casas ${ }^{1} \&$ Sergi Villalba ${ }^{2}$ \\ ${ }^{1}$ Technical University of Catalonia, UPC-BarcelonaTech. Department of Civil and \\ Environmental Engineering, c/ Jordi Girona 1-3, 08034 Barcelona, Spain \\ ${ }^{2}$ Technical University of Catalonia, UPC-BarcelonaTech. Department of Project and \\ Construction Engineering, c/ Colom 11, 08222 Terrassa, Spain
}

Corresponding author: Tel.: +34934016200 email: antonio.jose.de.sousa@upc.edu

\begin{abstract}
In this paper, the authors conducted an experiment where a reinforced concrete beam was instrumented with a 5-meter-long polyimide single DOFS performing four equal segments externally bonded to the bottom surface of the element, using for each segment a different type of adhesive. Three strain gauges were also used for comparison purposes. This beam was then loaded, producing expected equal levels of strain in each of the fiber segments for a more direct comparison of the different adhesives performance. The effect of alternating the spatial resolution is also analysed. In this exercise, additionally to the comparison with the other instrumented sensors it is also important the consideration and analysis of the associated Spectral Shift Quality (SSQ) values of the DOFS measurements.
\end{abstract}

Keywords: Distributed optical fiber sensors, Rayleigh backscatter, structural health monitoring, sensor bonding, reinforced concrete 


\section{Introduction}

The maintenance and safety of infrastructures is of great importance for the overall wellfunctioning of the modern society. All civil engineering infrastructures are subject to natural hazardous and ordinary mechanical resistance decay and deterioration through its lifetime. Therefore, the accessible and immediate maintenance of such infrastructures is of paramount importance for the safety of its users and the economic competitiveness of any region.

It is in this context that the research and development of viable and robust Structural Health Monitoring (SHM) techniques in the past decades has become an important tool for the increase of the service life of these infrastructures. The early and correct detection of the damage in these infrastructures derives on great economical savings, a better structural maintenance and an improved assessment of the structural safety.

Within SHM applications, the use of optical fiber sensors (OFS) has been a popular topic of research and practice in the last two decades. This is due to their distinctive properties that offer significant advantages when compared with the more conventionally used electric sensors. OFS are immune to electromagnetic interference presenting measurements with lesser noise. These sensors perform also well under a wide range of temperature variations and since they are chemically inert, they are also free from corrosion. Lastly, OFS are generally small and lightweight, which facilitates the transport and installation of such sensors in a wide range of application scenarios. The use of these sensors has been well documented and practiced particularly through the use of Fiber Bragg Grating sensors [1].

On the other hand, the particular study and use of distributed optical fiber sensors (DOFS) is a relatively more recent topic of research within civil engineering infrastructures SHM. At the same time that these sensors provide the same advantages of 
the aforementioned optical fiber sensors, with the additional clear advantage of being able of acquiring strain and/or temperature information for longer extensions of the monitored structural components on which they are deployed and with very high spatial resolution $[2]$.

This advantage is of great convenience and importance for the monitoring of concrete structures where the exact location of the possible crack formations is unknown beforehand and in this way, might be missed with the use of point or discrete sensors if not positioned at that particular location. With the use of distributed sensing, virtually every cross-section along the length of the fiber sensor is being monitored.

Furthermore, with the use of DOFS a larger area of the structure may be monitored with the use of up to one single sensor, which only requires one connecting cable, thus enabling a simpler and more economical monitoring system.

Previous work has displayed the promising capabilities of this technology on the monitoring of civil engineering infrastructures [3]-[8]

Nevertheless, as mentioned before, this is still a relatively new approach of this technology in the application on civil engineering infrastructures, which still presents substantial uncertainties regarding its use. Therefore, a continuous and deeper research of this topic is of vital importance for an eventually more systematically and regular use in SHM applications. 


\section{Distributed optical fiber sensing (DOFS)}

When using DOFS technology, the optical fiber cables can be bonded to the surface or embedded inside the material. When strain and temperature variations occur, these changes are transferred from the monitored material to the sensor which variates the scattered signal being reflected inside the fiber. As mentioned before, the use of DOFS, provide additional advantages when compared with the use of their discrete or point sensors counterpart. However, these advantages are also strongly correlated with the specific use of each of the scattering techniques, which enables distributed sensing.

Scattering is at the origin of distributed optical fiber sensing and is defined by the interaction between the light and the optical medium. From this interaction three different scattering processes occur, where each can be used for different applications due to their inherent particularities. These scattering processes are the Raman, Brillouin and Rayleigh scattering [2].

Raman scattering is highly dependent of temperature variations, which has led to some applications in civil engineering SHM but has been more typically used in other fields [5].

Brillouin scattering based DOFS on the other hand have been the most studied and used DOFS systems in civil engineering SHM applications due to their large-scale capability, which can go up to the kilometre range. This technique was initially introduced with the use of optical time domain reflectometry (OTDR) which has an inherent low spatial resolution (of around $1 \mathrm{~m}$ ), which is not ideal for crack detection applications. This was in the meantime improved through the development of the Brillouin optical time domain analysis (BOTDA) which relatively enhances the achievable spatial resolution, being currently more frequently used the Brillouin scattering technique. In fact, the largest ongoing real-life monitoring application of DOFS uses this technique as described in [9]. 
On the other hand, the optical frequency domain reflectometry (OFDR) systems, which are based in the Rayleigh scattering, offer an unprecedented spatial resolution, as high as one millimetre, which makes it ideal for crack detection and localization although currently limited to a monitoring length of $70 \mathrm{~m}$. This is achieved through the use of swept wavelength interferometry (SWI) to measure the Rayleigh backscatter as a function of length.

The Rayleigh backscatter, for any given fiber as a function of distance, is a random but static property working as an individual fingerprint for each specific cable. External stimulus to the fiber such as strain or temperature variations induce shifts in the locally reflected spectrum and in this way facilitating distributed strain or temperature sensing with a high spatial resolution [10].

In this way, in the present study it is used the ODiSI A model from LUNA technologies which is an optical backscattered reflectometry (OBR) system based on the Rayleigh OFDR, Figure 1. This system allows the acquisition of distributed strain and temperature measurements with sub-cm spatial resolution and a $\pm 2 \mu \varepsilon$ and $\pm 0.2{ }^{\circ} \mathrm{C}$ resolution. The maximum sensing length is of $50 \mathrm{~m}$ and the strain measurement range is of $\pm 13000 \mu \varepsilon[11]$.

More detailed information of this technology and the theoretical background of distributed optical fiber sensing can be consulted in [2]. 


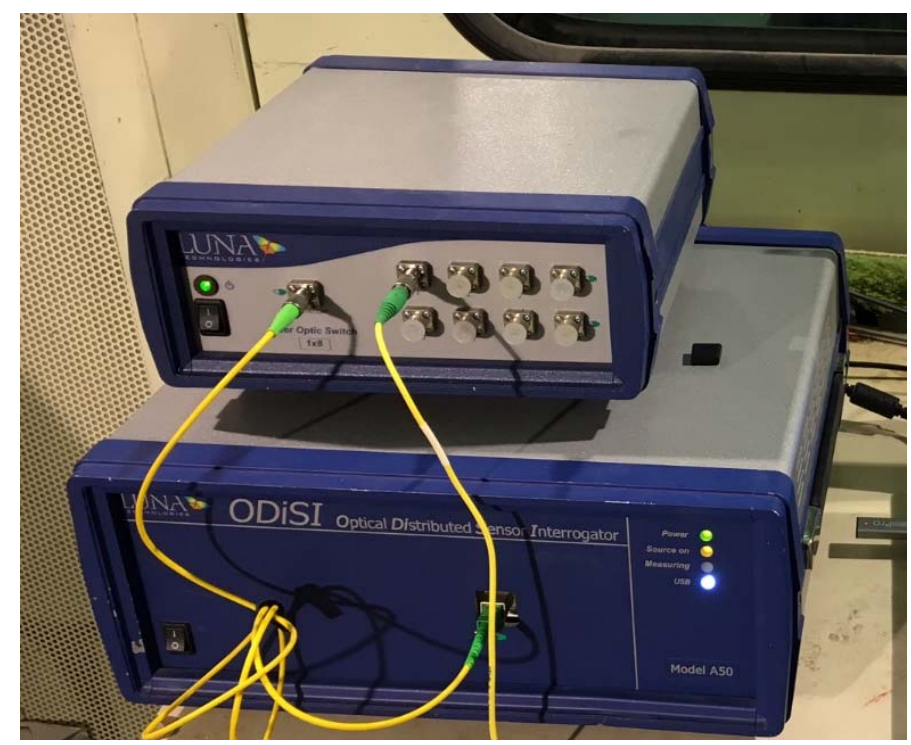

Figure 1. ODiSI A system used in the laboratory test campaign

\section{DOFS adhesive bonding performance analysis}

Despite the significant increase in interest on the study and practice of SHM applications through the use of DOFS, due to its relatively novelty, a considerable lack of knowledge is still present regarding the choice of the optimal bonding adhesive, especially in its use when deploying these sensors in reinforced concrete structures.

In order to assure a reliable monitoring of the strain data in the substrate, an optimal strain transfer between the sensor and the monitored material must be achieved, therefore the topic of its deployment is of considerable importance.

When using these sensors, a compromise exists regarding the strain measurements accuracy and the achievable protection and durability. It is easily understandable that the use of fibers with relatively thicker coatings provides better care to the sensor and eases the handle an manipulation at the time of installation but, at the same time, having a greater effect on the measured data as it decreases thes strain precision. On the other hand, a thinner coating creates the necessity of greater care when handling the sensors due its higher fragility but at the same time increases the effectiveness of the strain transfer between the sensor and substrate material. 
Additionally, different studies have been produced where special packaging schemes were developed in order to secure the durability and handling of DOFS, such as in [13].

In this research, the authors use a polyimide coated fiber that had a combined total diameter of core, cladding and coating of $155 \mu \mathrm{m}$ [12], being that the coating only contributed with $15 \mu \mathrm{m}$ for this diameter with the objective of reducing the influence of the sensor coating material in the strain accuracy of the DOFS measurements.

This is of relevance for reinforced concrete applications where the heterogeneity of the material presents additional challenges for the optimal bonding of the sensors.

In this way, in addition to the removal of any grease or dust present in the surface of the host material and the smoothening of the surface, the decision on the adhesive to bond the optical fiber sensor to the surface has also to be assessed and considered. Being this of even more importance when applying DOFS such as the case of the deployed fiber in this research, Figure 2.

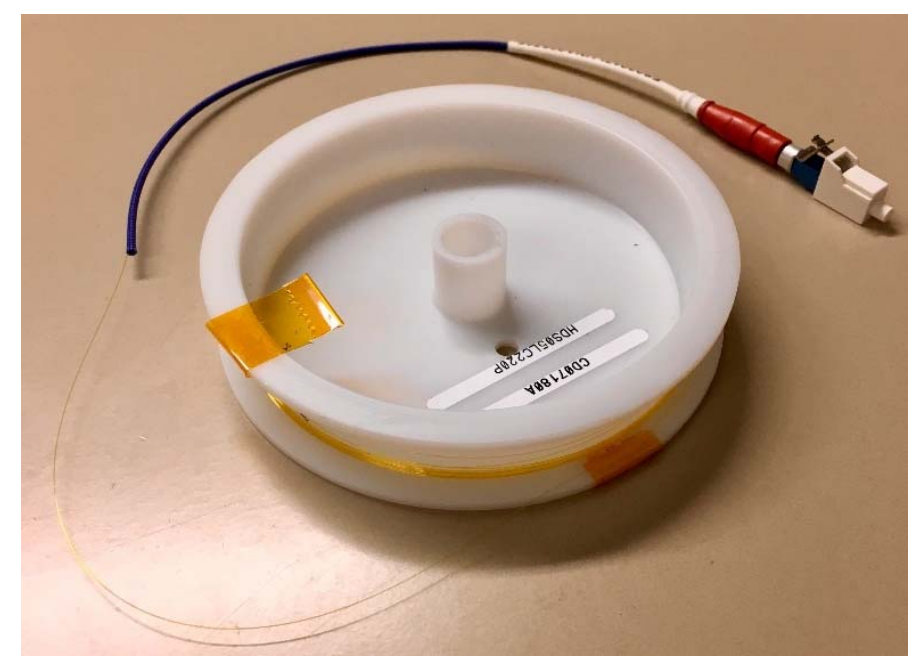

Figure 2. DOFS type deployed in the experimental campaign

There have been some studies where the coating influence on the strain transfer on point fiber optic sensors [14], the adhesive spread installation method [15] and the coating strain transfer impact in DOFS [16] were analysed. 
Moreover, one particular study [17], analysed among other things the performance of the use of cyanoacrylate and epoxy adhesives together with both nylon and polyimide DOFS when attached to the surface of the concrete and when attached to the reinforcement of the same structural elements. In this case, the results displayed a better performance of the polyimide fiber deployed together with the cyanoacrylate adhesive when bonded to the reinforcement and the combination of nylon fiber with the epoxy adhesive when bonded to the concrete.

Furthermore, in the authors personal experience, a combined use of epoxy adhesive with polyimide fiber when bonded to the concrete surface had offered promising results in different experiments [3], [5], [18].

Consequently, in the present paper, the authors decided to conduct a test where a single $5 \mathrm{~m}$ polyimide DOFS is attached to the surface of a reinforced concrete beam using four adhesives: epoxy, cyanoacrylate, polyester and neutral cure silicone, Figure 3.

These adhesives were selected based on the previous experience of the authors and through what was found during a literature review on this topic. Namely, the three first adhesives: epoxy, cyanoacrylate and polyester were ordered as advised by the TML strain gauges 2017 catalogue for concrete applications [19]. On the other hand, the used neutral cure silicone, was a simple commercial one from Ceys brand for general construction applications where concrete was included. 


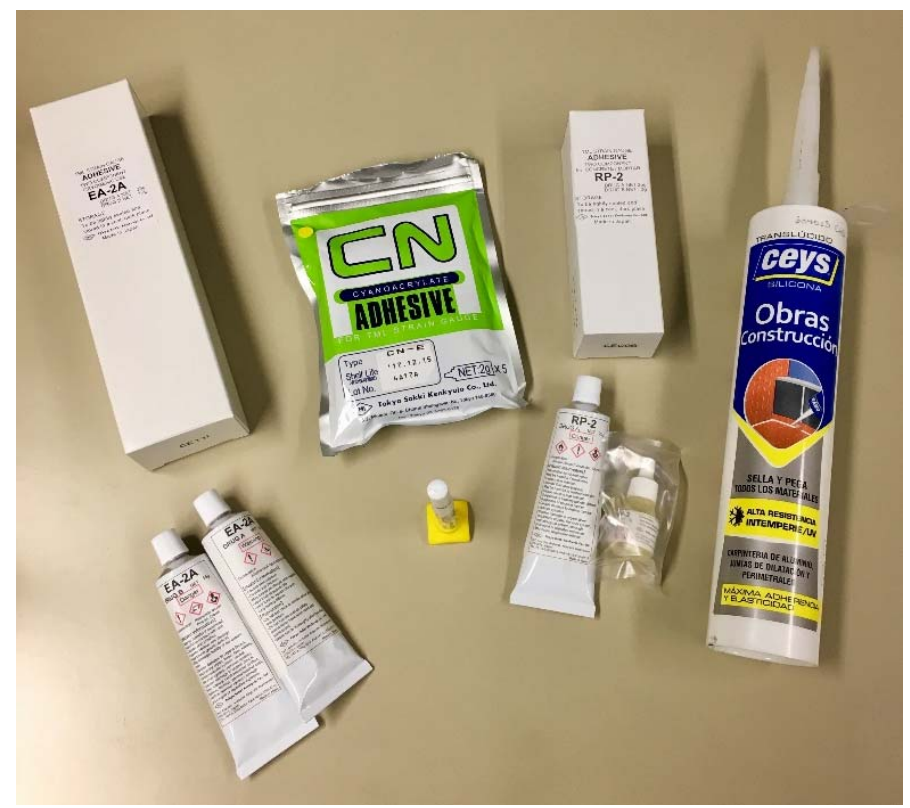

Figure 3. Adhesives deployed in this experimental campaign, from left to right: Epoxy, Cyanoacrylate, Polyester, Neutral Cure Silicone

It is important to mention that to the author's knowledge, the use of silicone in this type applications had only been performed before as an additional layer of protection of the sensor for the case of embedded implementations. In this case, for the first time the silicone is deployed primarily as an adhesive. In this sense, it is important to use a neutral cure silicone in order to not harm the polyimide fiber as it may occur when using an acetoxy cure one.

Finally, it has to be also referred that in practical terms it is important to have in consideration the viscosity and time of curing of each adhesive. In real world scenarios, it is often the case where the surface where the sensor is going to be instrumented is not horizontal or facing up. Therefore, for these situations it is important to deploy a very fast curing adhesive that allows for an also fast bonding. Moreover, the chosen adhesive should also have a high viscosity that prevents its dripping along the member which would weaken the bond. Each one of the chosen adhesives for this experiment provides different times of curing and viscosity options that have to be considered carefully depending on each intended application. 


\section{Experimental setup}

As mentioned before, the main goal of this experimental campaign was to assess the performance of different adhesives for the bonding of DOFS to concrete elements during a three-point load test.

A 60x15x15 [cm] reinforced concrete beam, Figure 4, was used. Furthermore, as observed in Figure 4, this structural element had two longitudinal $\phi 12$ and four $\phi 6$ S500 rebars.
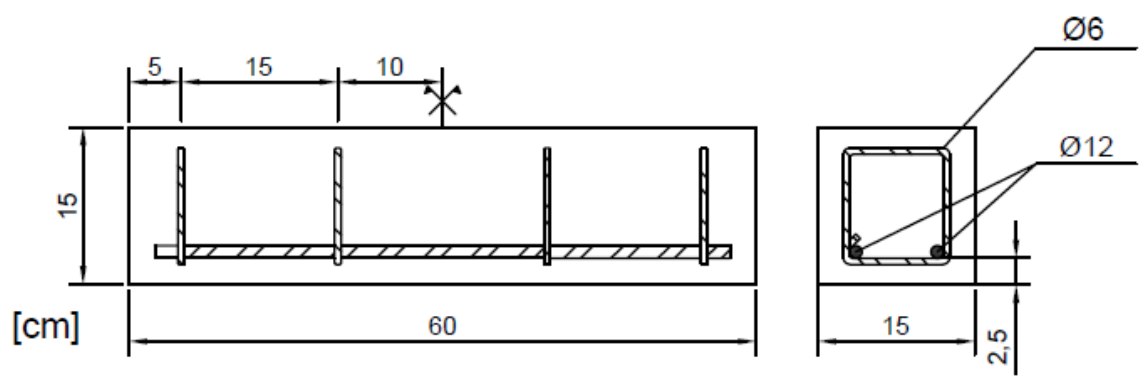

Figure 4. Tested beam definition scheme

A $5.2 \mathrm{~m}$ long DOFS was bonded to this concrete by providing a pattern with four different equal segments on the bottom surface of the beam. This was done with the intention to submit them to the same strains (in tension) and therefore they would be directly and immediately comparable.

Additionally, three $30 \mathrm{~mm}$ length strain gauges were also bonded to the centre of the bottom surface of the concrete beam with equal distance between them: SG1, SG2 and SG3. These sensors are later used for comparison purposes with the data obtained by the DOFS, Figure 5. The deployment of the instrumented sensors on the reinforced concrete beam with the information of the adhesive used in each DOFS segment is presented in Figure 5. A photo of the specimen is also seen in Figure 6. 
BEAM - BOTTOM VIEW

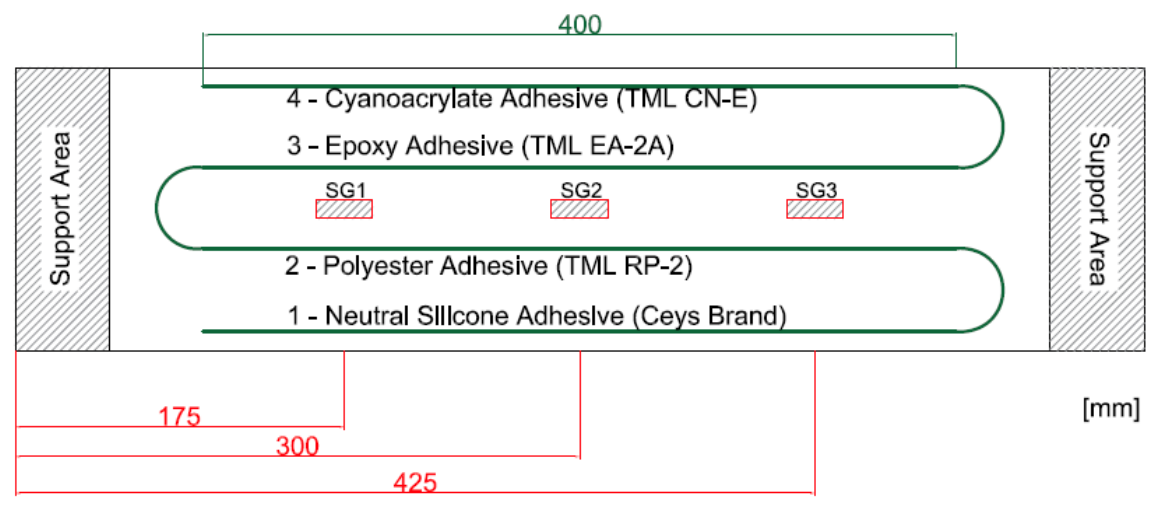

Figure 5. Plan of the reinforced concrete beam instrumented sensors

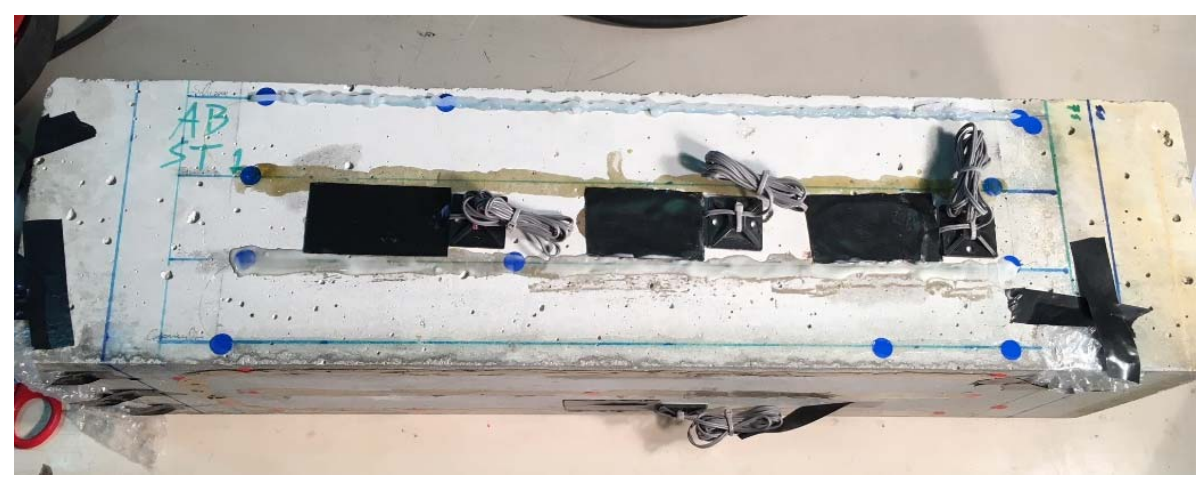

Figure 6. View of the sensors (DOFS and strain gauges) instrumented in the bottom surface of the tested beam

In this way, the global setup of the three-point load test on this beam can be seen in Figure 7. 


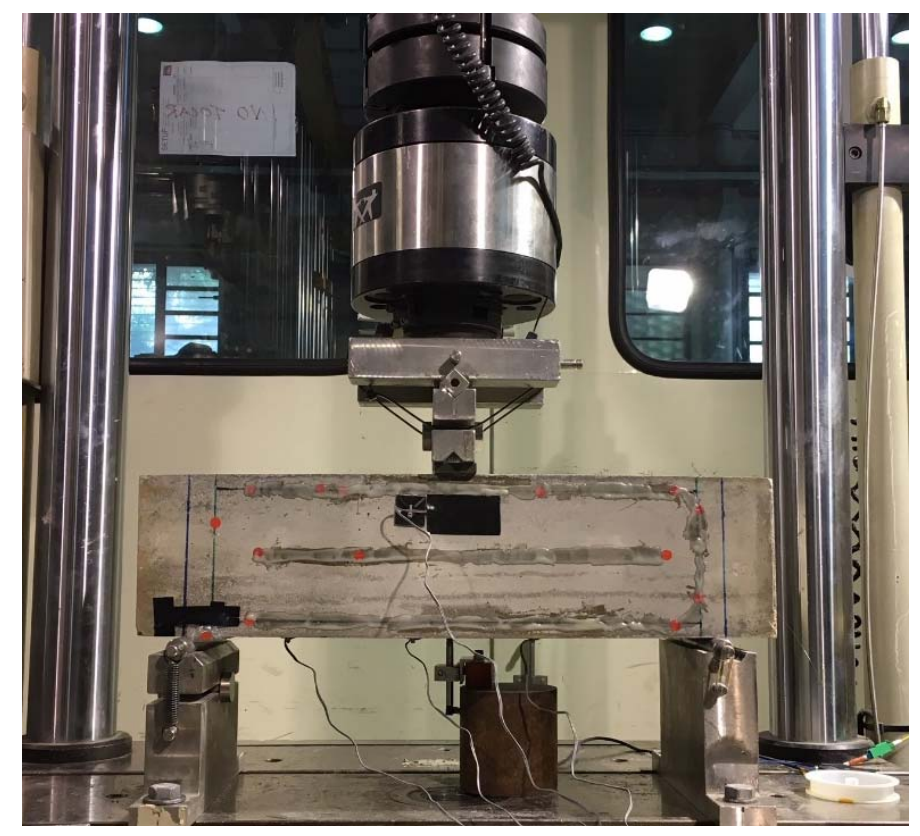

Figure 7. View of the loading arrangement (the DOFS segments considered in the analysis are the ones on the bottom surface of the beam)

At the time of pouring the concrete, additional cylindrical samples were also produced. Afterwards, close to the date of the test, these samples were tested in order to calculate the material properties of the used concrete. Therefore, the mean compressive strength $\left(f_{c m}\right)$, the mean tensile strength $\left(f_{c t m}\right)$ and the mean Young modulus $\left(E_{c}\right)$ were obtained. Consequently, with this information, it is also possible to calculate the strain level expected to initiate the formation of cracking at the concrete $\left(\varepsilon_{c t}\right)$. These properties are described in Table 1.

Table 1. Concrete material properties

\begin{tabular}{c|c|c|c|c}
\hline Properties & $\begin{array}{c}\boldsymbol{f}_{\boldsymbol{c m}} \\
{[\mathbf{M P a}]}\end{array}$ & $\begin{array}{c}\boldsymbol{f}_{\boldsymbol{c t m}} \\
{[\mathbf{M P a}]}\end{array}$ & $\begin{array}{c}\boldsymbol{E}_{\boldsymbol{c}} \\
{[\mathbf{M P a}]}\end{array}$ & $\begin{array}{c}\boldsymbol{\varepsilon}_{\boldsymbol{c t}} \\
{[\boldsymbol{\mu \varepsilon}]}\end{array}$ \\
\hline Specimen & 48.027 & 3.944 & 37886.64 & 104.10 \\
\hline
\end{tabular}

An additional point of interest in this study was the assessment of the influence of different spatial resolution used in the DOFS system. Here the objective was to observe if a milimetric spatial resolution would improve significantly the strain monitoring on 
concrete members compared with a resolution in the centimetre level. It is important to mention that milimetric spatial resolution level implies ten times the amount of acquired data. Furthermore, due to the heterogeneity of the concrete material and its aggregate sizes, this aspect is of interest for the application of DOFS based monitoring systems in this material.

In this way, it was decided to initially perform three separate but identical load cycles where a different spatial resolution was used. The maximum applied load in these cycles was only $11 \mathrm{kN}$, therefore not inducing cracking in the concrete.

Afterwards, a final load was applied inducing cracking and continuing until failure of either the specimen or the DOFS. The test sequence is defined in

Table 2 and Figure 8.

Table 2. Test cycle description

\begin{tabular}{c|c|c}
\hline Test cycle number & Spatial Resolution & \multirow{2}{*}{ Sampling Rate } \\
\hline 1 & $1 \mathrm{~cm}$ & \\
\hline 2 & $3 \mathrm{~cm}$ & \multirow{2}{*}{$0.2 \mathrm{~Hz}$} \\
\hline 3 & $1 \mathrm{~mm}$ & \\
\hline $4-$ rupture & $1 \mathrm{~cm}$ & \\
\hline
\end{tabular}
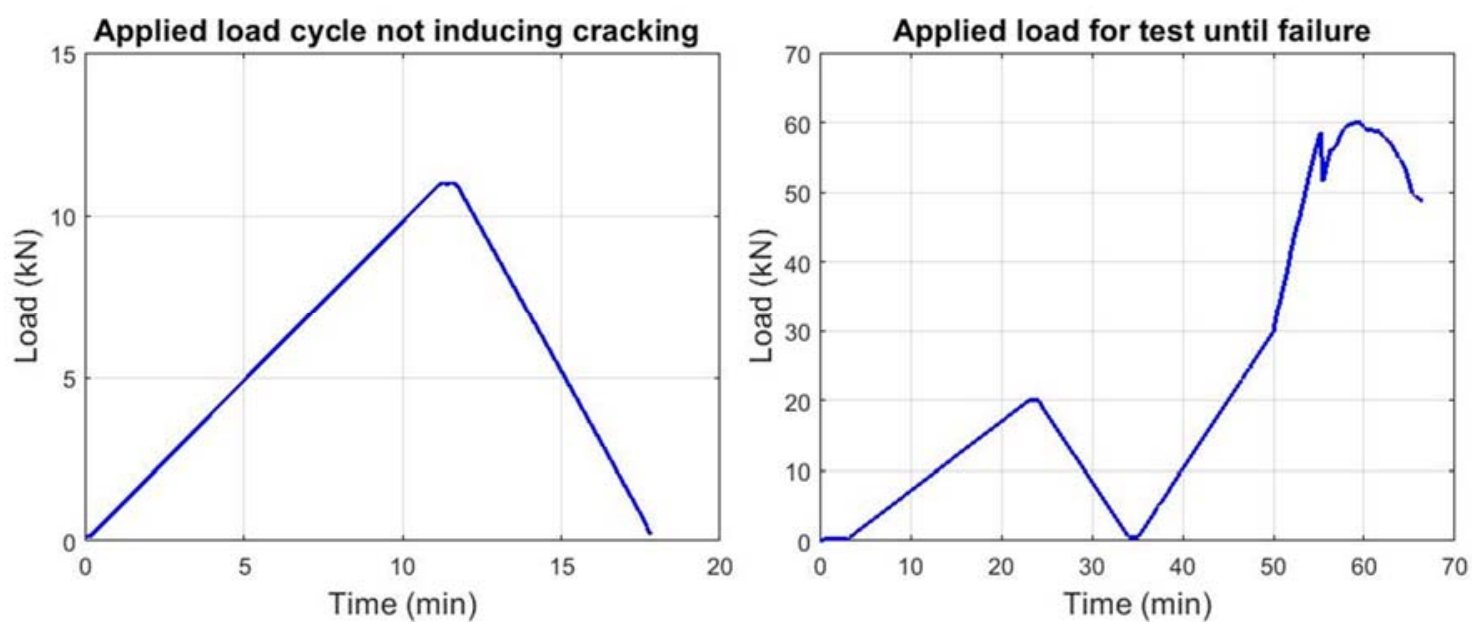

Figure 8 . Load cycle applied for tests 1 to 3 (left) and test 4 - rupture (right) 


\section{Results}

The data from both the DOFS and the strain gauges was post processed and analysed. The DOFS handling software allows the storage of the data in three different formats, namely: its raw spectral shift form, directly converted to strain/temperature using the manufacturer calibrated constant factors and finally in its associated Spectral Shift Quality (SSQ) matrix. This last one is of significant importance in order to assess the performance of the fiber during the test, especially after the occurrence of damage (cracking).

\subsection{Comparison of spatial resolution input}

As described in Error! Reference source not found., it was decided to input three different spatial resolutions of the DOFS measurements in the first three load cycles applied to the tested specimen: $1 \mathrm{~cm}, 3 \mathrm{~cm}$ and $1 \mathrm{~mm}$. The $3 \mathrm{~cm}$ spatial resolution is justified by the minimum length of the available strain gauges recommended in concrete measurements. Thus, 520 points, 174 points and 5191 points of the DOFS were measured in the first, second and third load test cycle respectively.

In Figure 9, the obtained strain measurements for the different used adhesives and inputted spatial resolutions for a load of $11 \mathrm{kN}$ are displayed. The results from the strain gauges is also displayed for comparison 

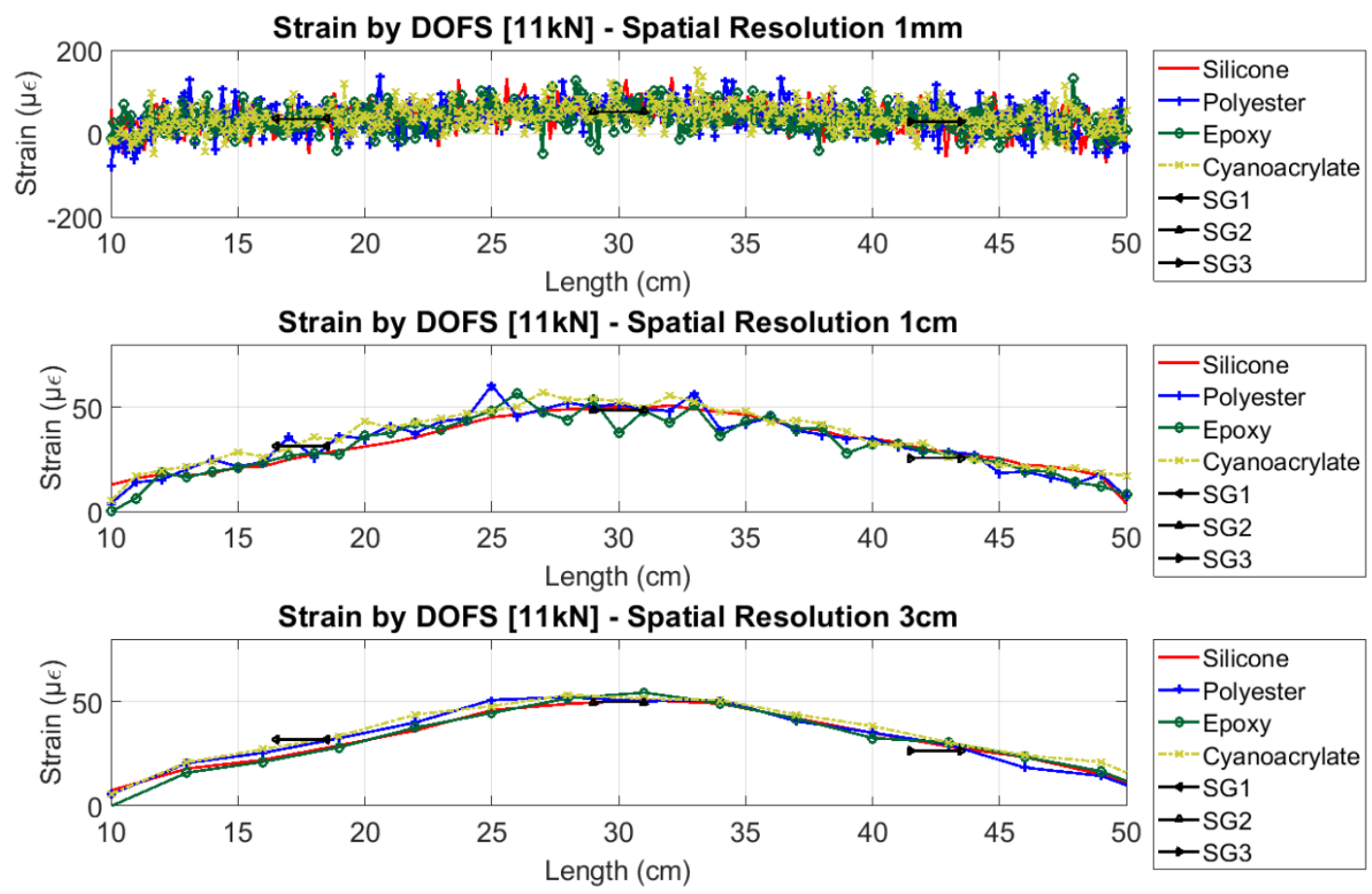

Figure 9. Strain data measured by DOFS with different spatial resolution inputs and adhesives.

As it is possible to observe from this figure, there is a clear difference between the data measured using a $1 \mathrm{~mm}$ and the two spatial resolutions, presenting a significantly higher spatial variability. This is due to the heterogeneity of the sizes of the aggregates that constitute the concrete material. A still relatively noticeable variability is observed for the $1 \mathrm{~cm}$ spatial resolution (except for the silicone bonded segment) but significantly smaller than the one verified with $1 \mathrm{~mm}$ and that presents a good agreement with the strain gauges and the measurements observed with a $3 \mathrm{~cm}$ spatial resolution.

This is also validated by observing the box plot of the calculated difference between the three spatial resolutions data sets as seen in Figure 10 and Table 3. Here it is seen that the $1 \mathrm{~mm}$ data set presents significant higher difference bot compared with the $1 \mathrm{~cm}$ and $3 \mathrm{~cm}$ data set. All calculated mean differences are very close to zero being $-1.07 \mu \varepsilon,-1.23 \mu \varepsilon$ and $0.29 \mu \varepsilon$ for $\Delta(1 \mathrm{~mm}-3 \mathrm{~cm}), \Delta(1 \mathrm{~mm}-1 \mathrm{~cm})$ and $\Delta(1 \mathrm{~cm}-3 \mathrm{~cm})$, respectively. 


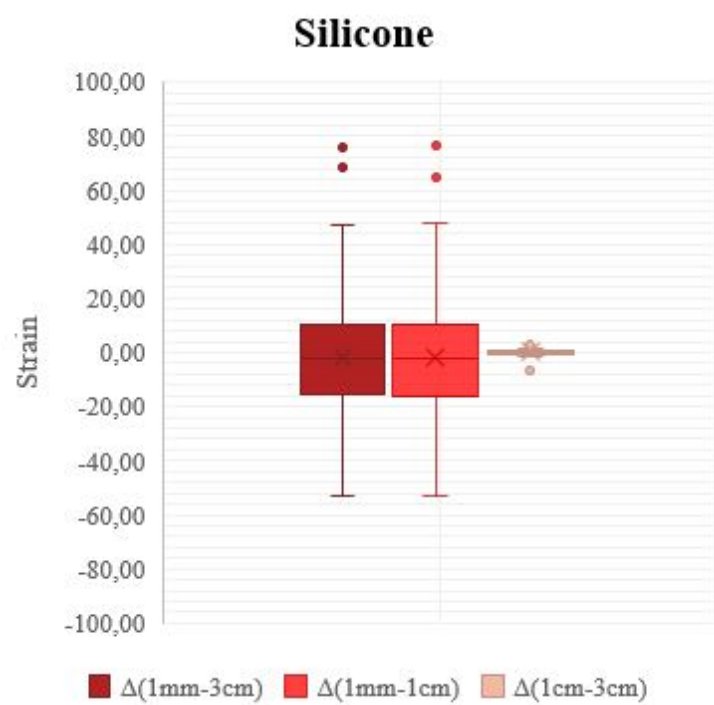

Epoxy

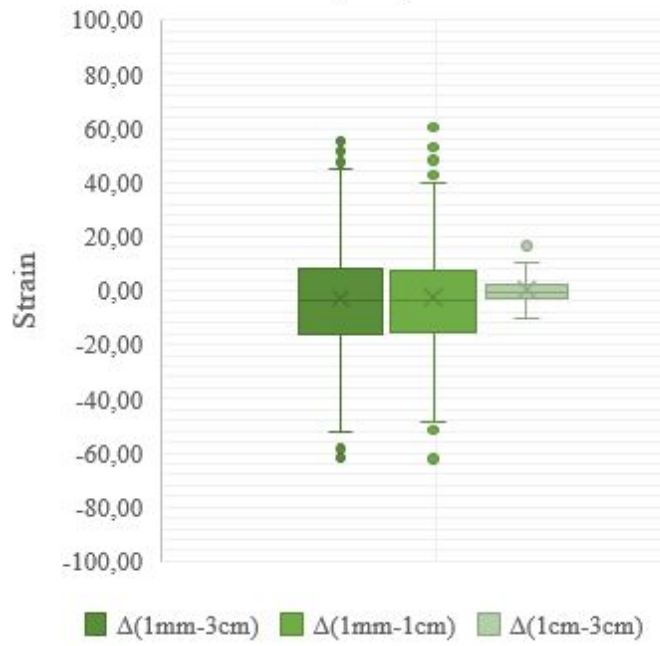

\section{Polyester}

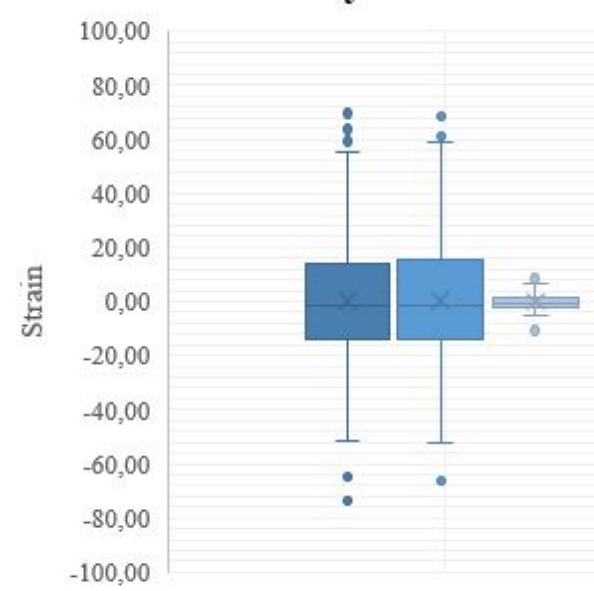

$\Delta(1 \mathrm{~mm}-3 \mathrm{~cm}) \square \Delta(1 \mathrm{~mm}-1 \mathrm{~cm}) \square \Delta(1 \mathrm{~cm}-3 \mathrm{~cm})$

Cyanoacrylate

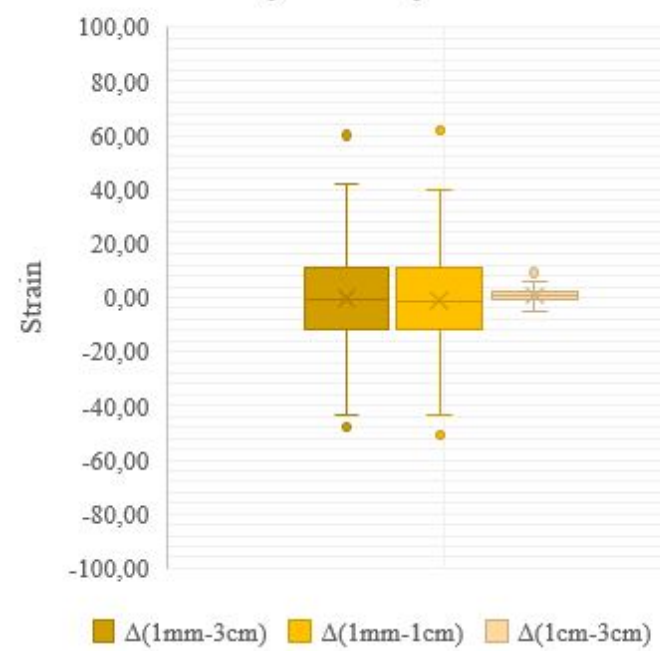

Figure 10. Box plot of the calculated difference between different used spatial resolutions for each bonded segment

Table 3. Calculated mean and standard deviation of differences between the different used spatial resolutions measurements [All units represent microstrain $(\mu \varepsilon)$ ]

\begin{tabular}{|c|c|c|c|c|c|c|}
\hline & & Silicone & Polyester & Epoxy & Cyanoacrylate & Average \\
\hline \multirow{2}{*}{$\Delta(1 \mathrm{~mm}-3 \mathrm{~cm})$} & mean $(\mu)$ & -1.65 & 0.47 & -2.99 & -0.11 & -1.07 \\
\hline & $\operatorname{std}(\sigma)$ & 19.18 & 22.03 & 19.62 & 15.94 & 19.19 \\
\hline \multirow{2}{*}{$\Delta(1 \mathrm{~mm}-1 \mathrm{~cm})$} & mean $(\mu)$ & -1.93 & 0.43 & -2.6 & -0.83 & -1.23 \\
\hline & $\operatorname{std}(\sigma)$ & 19.25 & 22.42 & 19.42 & 16.26 & 19.34 \\
\hline \multirow{2}{*}{$\Delta(1 \mathrm{~cm}-3 \mathrm{~cm})$} & mean $(\mu)$ & 0.24 & -0.02 & 0.19 & 0.76 & 0.29 \\
\hline & $\operatorname{std}(\sigma)$ & 1.59 & 3.82 & 5.03 & 3.08 & 3.38 \\
\hline
\end{tabular}


Notwithstanding, the standard deviation of these differences is clearly higher between the $1 \mathrm{~mm}$ and both the $1 \mathrm{~cm}$ and $3 \mathrm{~cm}$ data sets, being of $19.19 \mu \varepsilon$ for $\Delta(1 \mathrm{~mm}-3 \mathrm{~cm}), 19.34 \mu \varepsilon$ for $\Delta(1 \mathrm{~mm}-1 \mathrm{~cm})$ whereas it is only of $3.38 \mu \varepsilon$ for $\Delta(1 \mathrm{~cm}-3 \mathrm{~cm})$.

Having this in mind, it was decided to assess if even initially inputting a random spatial resolution it would be possible to obtain equal or similar results as using a different resolution. This is done by means of performing a moving average that would represent the desired resolution.
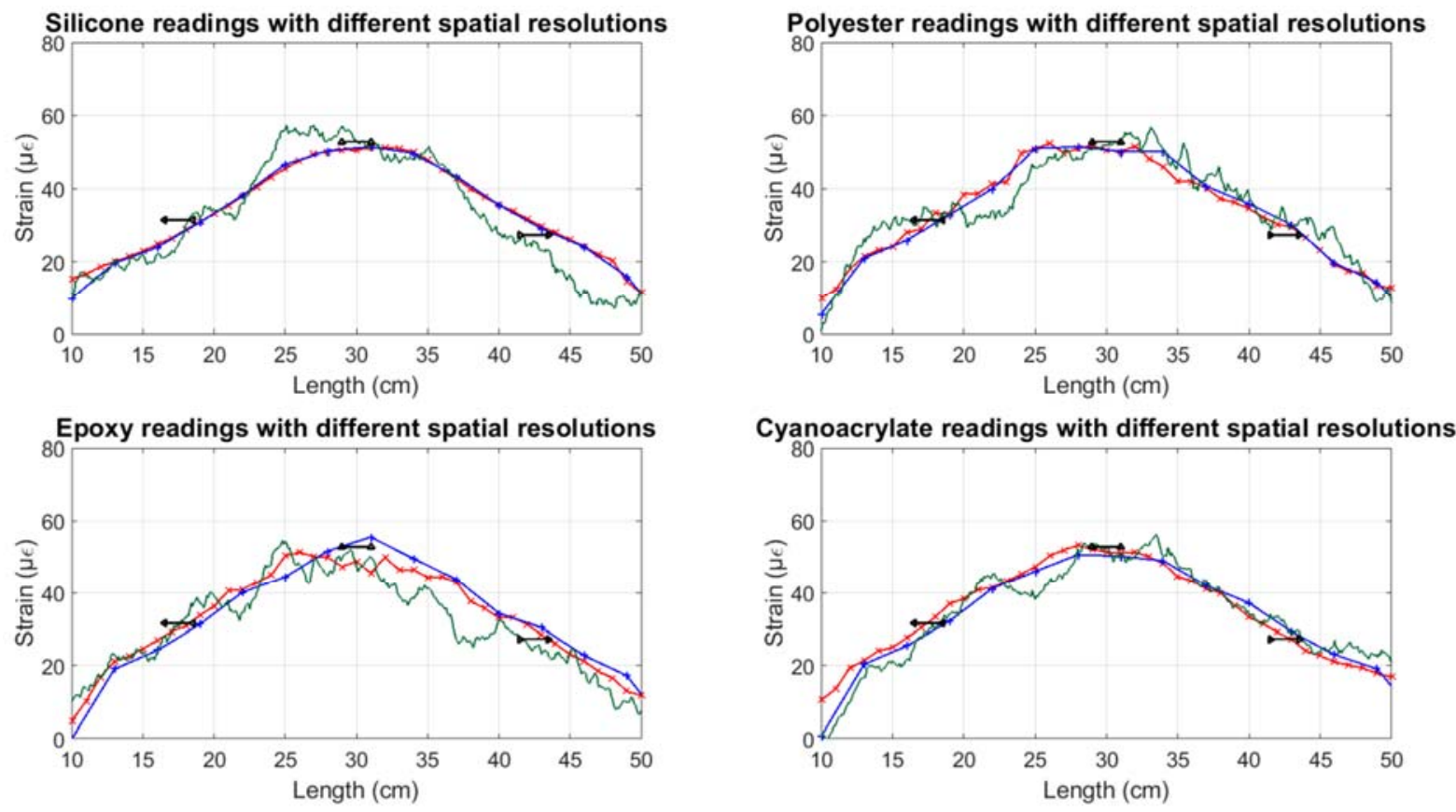

$\rightarrow-1 \mathrm{~cm}$ with MAvg of $3 \rightarrow 3 \mathrm{~cm}-1 \mathrm{~mm}$ with $\mathrm{MAvg}$ of $30 \rightarrow-\mathrm{SG} 1 \rightarrow \mathrm{SG} 2 \rightarrow-\mathrm{SG} 3$

Figure 11. Data measured by each DOFS adhesive segment with different spatial resolutions while performing a moving average to the $1 \mathrm{~mm}$ and $1 \mathrm{~cm}$ data in order to directly compare it with the $3 \mathrm{~cm}$ spatial resolution data

In this way, the measurements obtained with a $3 \mathrm{~cm}$ spatial resolution were directly compared with the other two sets by conducting a moving average with a window of 30 units to the $1 \mathrm{~mm}$ measurements and another with a window of 3 for the $1 \mathrm{~cm}$. The obtained graphs are depicted in Figure 11.

It is possible to observe how the averaged $1 \mathrm{~mm}$ obtained data improved significantly from before resembling the data from the other sets and the strain gauges 
but still presenting a noticeable erratic spatial variability. It is also perceived that apart the epoxy bonded DOFS segment, the set of averaged $1 \mathrm{~cm}$ data fits almost completely the one obtained using an initial $3 \mathrm{~cm}$ spatial resolution. Again, this is also observed through the box plot of the calculated differences between the different data sets as seen in Figure 12 and Table 4.

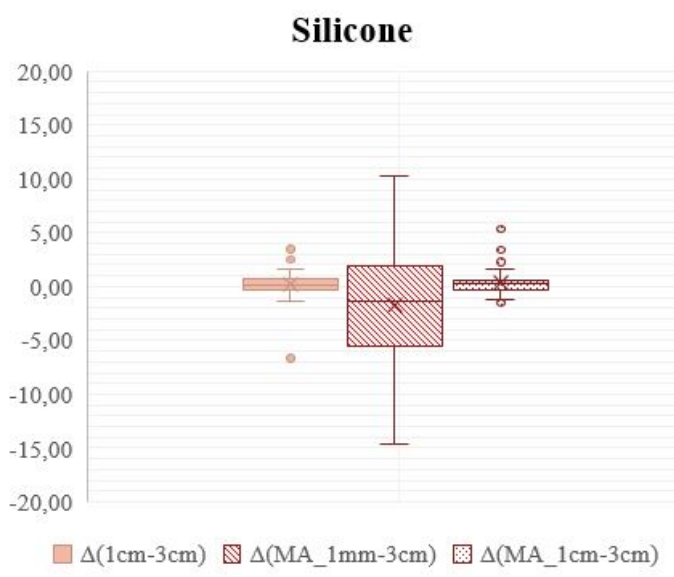

Epoxy

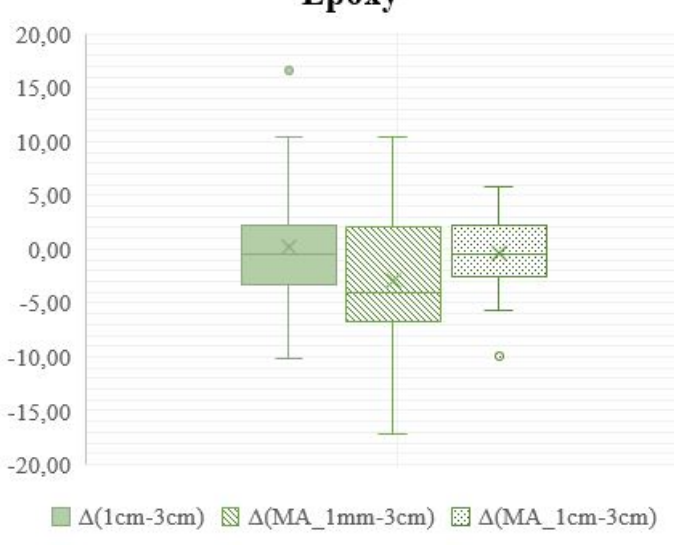

Polyester

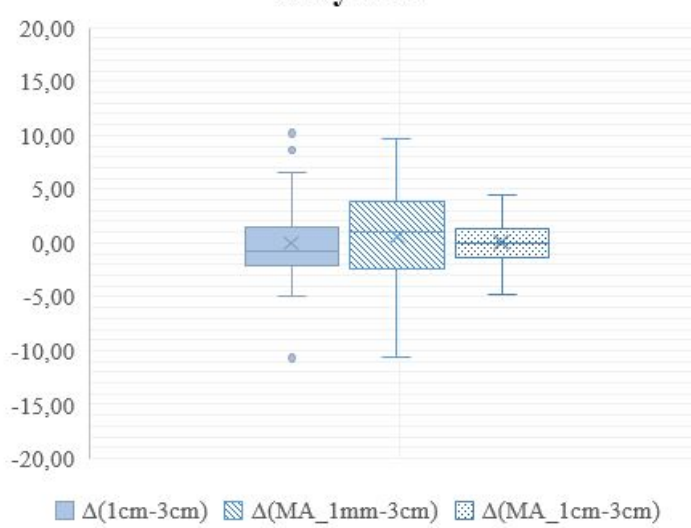

Cyanoacrylate

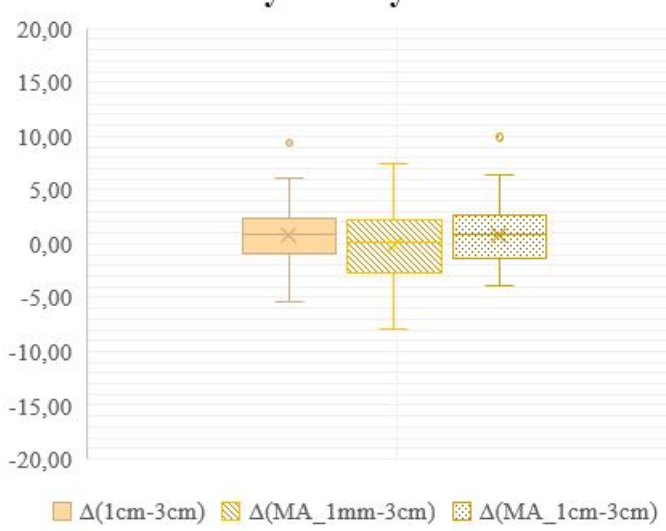

Figure 12. Box plot of the calculated difference between the data sets obtained through moving average and the $3 \mathrm{~cm}$ data set

Table 4. Calculated mean and standard deviation of differences between the different data sets after performing moving average [All units represent microstrain $(\mu \varepsilon)$ ]

\begin{tabular}{c|c|c|c|c|c|c}
\cline { 3 - 7 } \multicolumn{2}{c}{ Silicone } & Polyester & Epoxy & Cyanoacrylate & Average \\
\hline \multirow{2}{*}{$\begin{array}{c}\text { MA_1mm- } \\
\text { 1cm) }\end{array}$} & mean $(\boldsymbol{\mu})$ & -1.71 & 0.58 & -2.96 & -0.10 & $\mathbf{- 1 . 0 5}$ \\
\cline { 2 - 7 } std $(\boldsymbol{\sigma})$ & 5.41 & 4.26 & 6.20 & 3.08 & $\mathbf{4 . 7 4}$ \\
\hline \multirow{2}{*}{$\begin{array}{c}\text { MA_1cm- } \\
\text { 3cm) }\end{array}$} & mean $(\boldsymbol{\mu})$ & 0.31 & 0.08 & -0.35 & 0.82 & $\mathbf{0 . 2 2}$ \\
\cline { 2 - 7 } & std $(\boldsymbol{\sigma})$ & 1.17 & 1.87 & 3.35 & 2.84 & $\mathbf{2 . 3 1}$ \\
\hline
\end{tabular}


In Figure 12, the boxplot of $\Delta(1 \mathrm{~cm}-3 \mathrm{~cm})$ is again plotted in order to facilitate a direct comparison with the improvement made by the moving average routine. Here it is possible to see that, as mentioned before, despite a reduction of the standard deviation of $\Delta\left(\mathrm{MA} \_1 \mathrm{~mm}-1 \mathrm{~cm}\right)$ to $4.74 \mu \varepsilon$ compared to $19.19 \mu \varepsilon$ of $\Delta(1 \mathrm{~mm}-3 \mathrm{~cm})$, this value is still slightly higher than the calculated differences between the $3 \mathrm{~cm}$ data set and both the original $1 \mathrm{~cm}$ as well as the the MA_1 $\mathrm{cm}$ (after performing moving average) data sets.

Therefore, the conclusion is that no real advantages are originated by using a sub-cm spatial resolution. Furthermore, due to good agreement between the averaged $1 \mathrm{~cm}$ and $3 \mathrm{~cm}$ sets, it is decided to use the $1 \mathrm{~cm}$ spatial resolution, which provides a greater flexibility and better results considering the spatial resolution-accuracy-variability trade-off.

\subsection{Comparison of different adhesives under elastic loading behaviour}

The test cycles 1 to 3 are also used to analyse the performance of the different adhesives used to bond the DOFS to the concrete surface under non-cracked conditions.

In Figure 13 the strain measurements obtained through strain gauge 1 (SG1), strain gauge 2 (SG2) and strain gauge 3 (SG3) are compared with the corresponding points of each adhesive bonded segment of the DOFS. Since the deployed strain gauges have a $3 \mathrm{~cm}$ length, an average of the points of the DOFS, which are located at the same position of the length of the strain gauges is performed in order to more correctly compare both set of sensors. The applied load is also plotted using the right y-axis. A good agreement between the data from the DOFS and the strain gauges is observed.

At mid-span, where the strains are higher it is seen on Figure 13 and Figure 14 how apart from a small difference of $5.54 \mu \varepsilon(11.70 \%)$ of the epoxy bonded DOFS segment to SG2 all other adhesives have a difference around or below $2 \mu \varepsilon$. This corresponds to the resolution of the used DOFS acquisition system as mentioned before. 
Furthermore, as seen in Figure 14, all adhesives bonded DOFS segments present an average difference at the three locations below $13 \%$ which goes in agreement with what was verified in a previous test where a similar DOFS was compared with foil gauge strain sensors at different locations of a wind turbine blade [20].
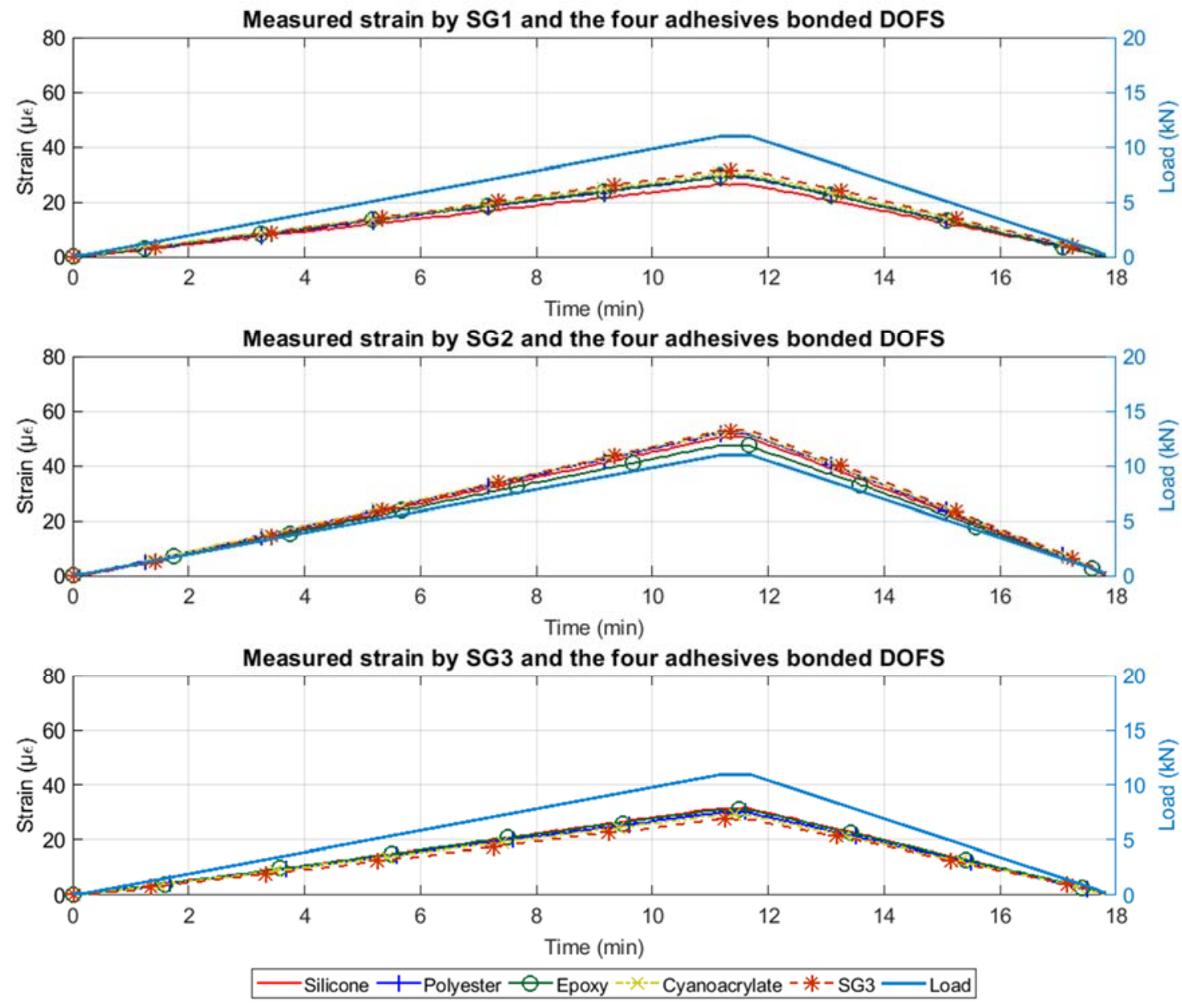

Figure 13. Comparison of strain measured by DOFS and SG1, SG2, SG3 with inputted load

Nevertheless, when assessing the overall distribution of the DOFS measurements for each segment over time, the surface plot of Figure 15 is obtained. Here it is seen a slightly smoother and homogenous reading at the neutral cure silicone segment when compared with the other adhesives by means of clearer increase of the contour lines of its surface plot. 


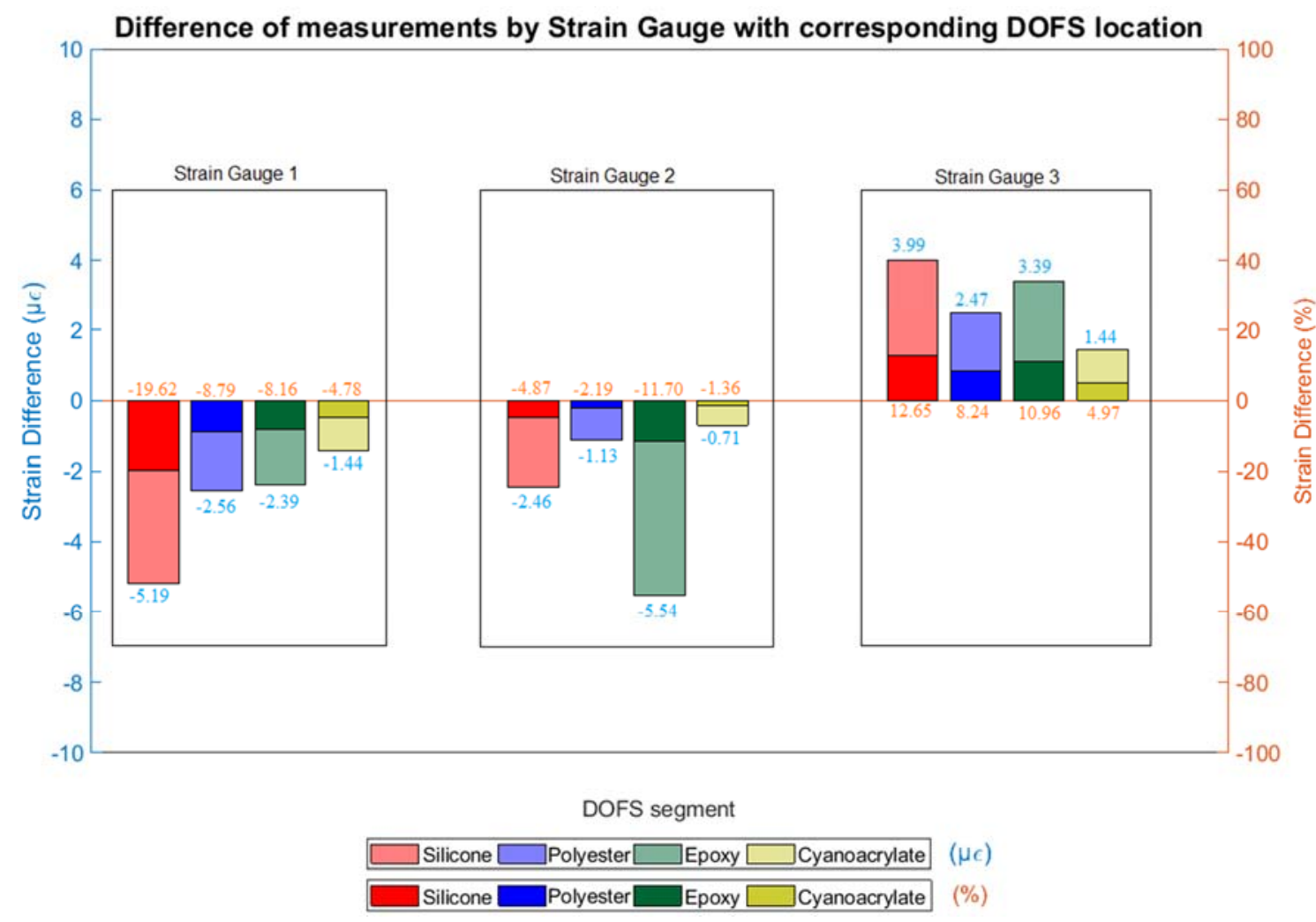

Figure 14. Strain difference of measurements by each DOFS bonded segment to corresponding Strain Gauge

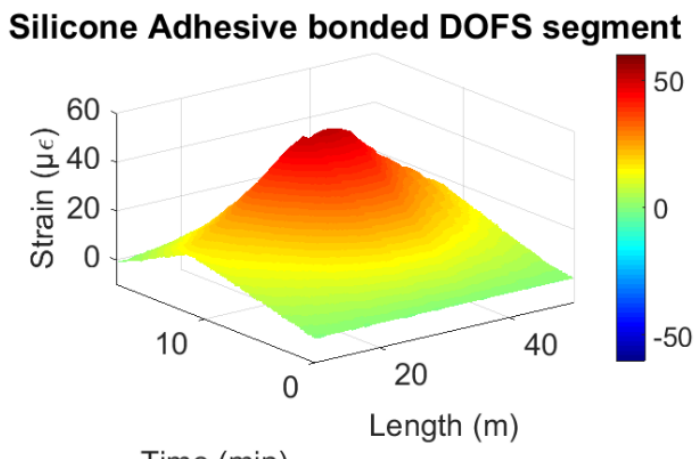

Time (min)
Polyester Adhesive bonded DOFS segment

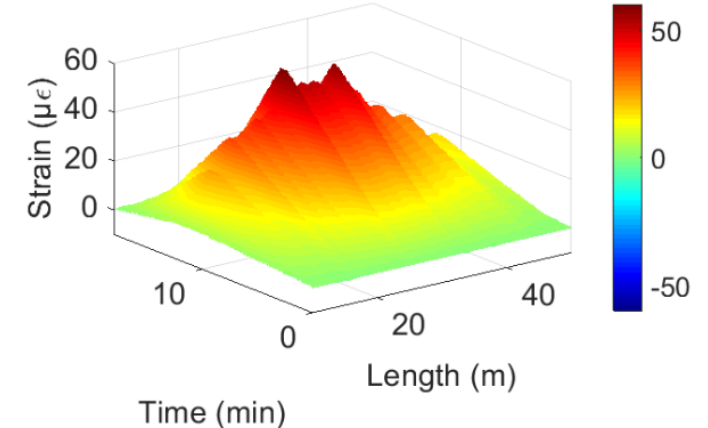

Epoxy Adhesive bonded DOFS segment Cyanoacrylate Adhesive bonded DOFS segment

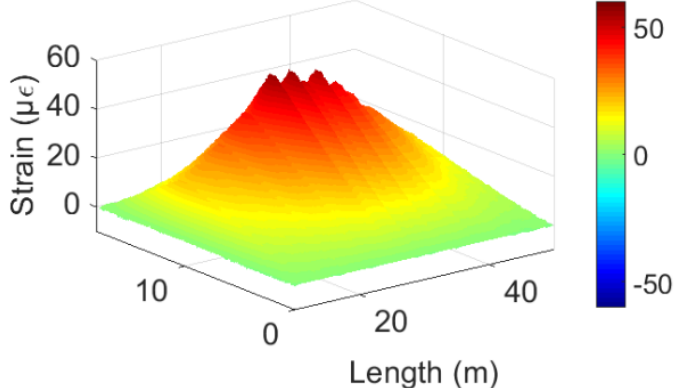

Time $(\min )$

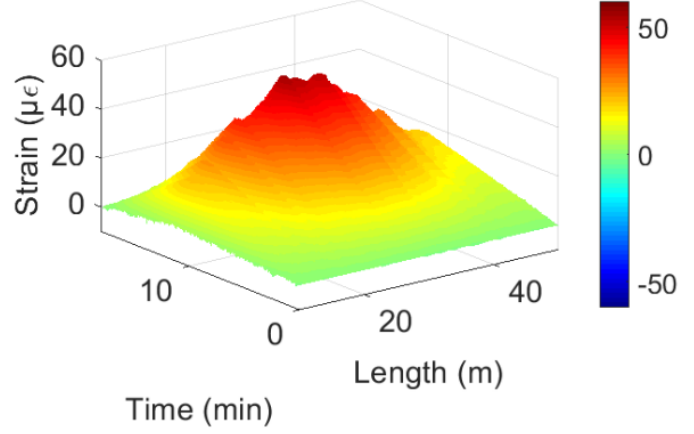

Figure 15. Strain measured for elastic load test cycle with different adhesive bonded DOFS segments 
The other used adhesives - Polyester, Epoxy and Cyanoacrylate - display a significant higher spatial variability especially at the point corresponding to the maximum strain value.

\subsection{Comparison of different adhesives when inducing cracking}

After conducting the load test cycles under non-cracked conditions a load test cycle inducing cracking was performed as depicted in Figure 8. Here, as mentioned before, the load was applied initially up to $20 \mathrm{kN}$, then decreased to zero and increased again until failure.

According to the mechanical properties obtained through the tests conducted to the cylindrical specimens and presented in Table 1, the cracking load would be close to $18 \mathrm{kN}$. Therefore, in the test, it was also desired to assess the performance of the different adhesives after cracking during the unloading stage. Nevertheless, during the execution of the load cycle it was verified that, the resistance of the beam specimen was relatively higher from what was expected.

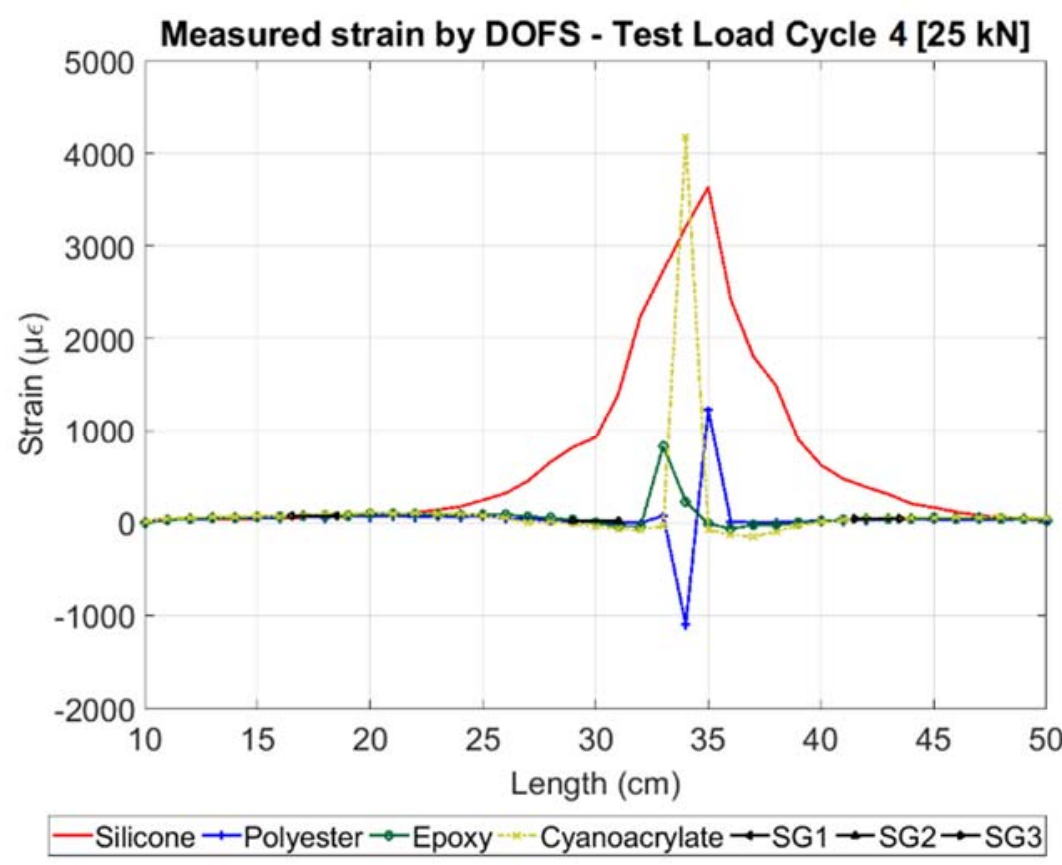

Figure 16. Detection and localization of cracking at concrete by DOFS 
In reality, as seen in Figure 17, cracking only started to occur around the $24 \mathrm{kN}$ load, being firstly detected by the silicone and cyanoacrylate bonded segments. In Figure 16 is displayed the detection of cracking by all segments at load level of $25 \mathrm{kN}$. Figure 17 depicts the evolution of the strain measurements by the four different adhesives bonded segments for different load stages and compared with the data obtained by the strain gauges.
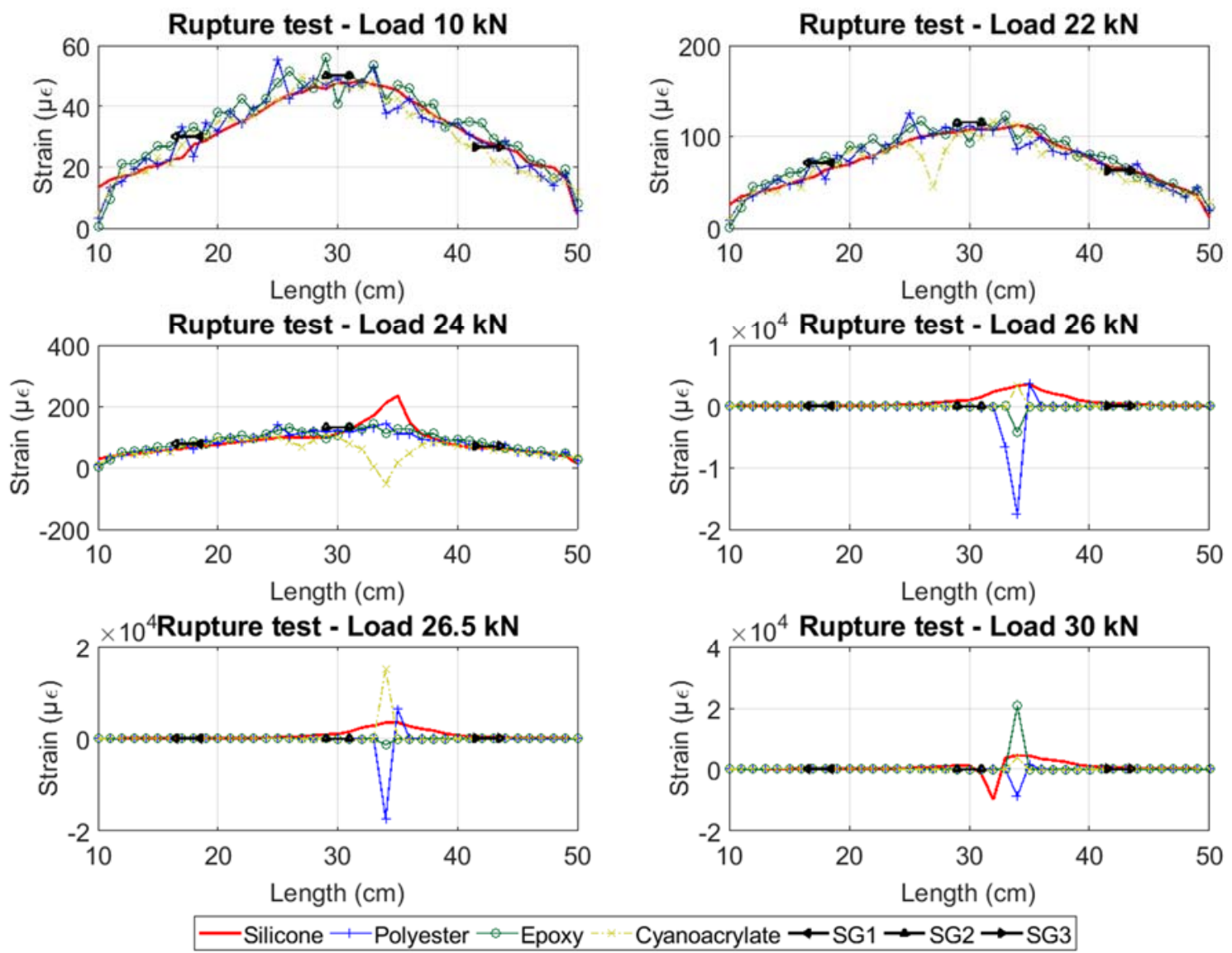

Figure 17. Evolution of the measured strain for different load levels until failure

As seen for the first part of the test (under un-cracked condition), before the occurrence of cracking, the silicone bonded segment presents significantly smoother spatial measurements when compared with the other adhesives.

After the initiation of cracking, all segments detect this damage at relatively the same location. This is in accordance to what was observed visually as the developed crack was not formed in a straight line orthogonal to the bonded DOFS segments. 
Afterwards, once the cracking occurs, all segments, apart from the silicone bonded one, start producing peak values with either positive or negative strain for subsequent load levels at the crack location. According to the loading sequence, only positive growing peaks should be observed.

This is related with the aforementioned Spectral Shift Quality (SSQ) values, which are associated with the DOFS measurements during the crack formation. This parameter is a quantitative measure of the correlation between the conducted measurement and the original baseline reflected spectra. Therefore, its value should theoretically be always between 0 and 1 . As mentioned before, the system manufacturer suggests the consideration of SSQ values below 0.15 as inaccurate, being that these values correspond to a change in strain or temperature that has likely exceeded the sensor measurable range [21].

Although the silicone bonded segment does not follow this trend, it should be highlighted that it presents, on the other hand, a significantly wider area around the location of the originated crack. This make it impractical to detect further cracks within this segment in the event of the fiber not being broken at that stage.

By analysing the measured strain the associated SSQ matrix, Figure 18 is obtained. It is observable how for all segments, a sharp and sudden change is detected both by the strain measurements and the SSQ matrix values at minute 47.33 , which corresponds to a load level of $24.65 \mathrm{kN}$. Slightly afterwards, the cracking initiation is detected by the silicone and cyanoacrylate segments (see Figure 17). The used fiber only ruptured at minute 62 for a load of $58.06 \mathrm{kN}$ and after the yielding of the tested specimen. Nevertheless for all the DOFS segments bonded to the bottom surface of the concrete and being analysed here, all measured points presented an SSQ value bellow 0.15 shortly after minute 50 for a load of $31.1 \mathrm{kN}$, becoming at this point effectively unusable. 
Moreover, it is possible to also observe in Figure 18 that, apart from the silicone bonded DOFS segment, before minute 47.33 there are some segment points, which display a relative smaller SSQ value than what is verified for the majority of the length of the fiber but also above the suggested 0.15 threshold. This happens close to the mid-span of the beam.
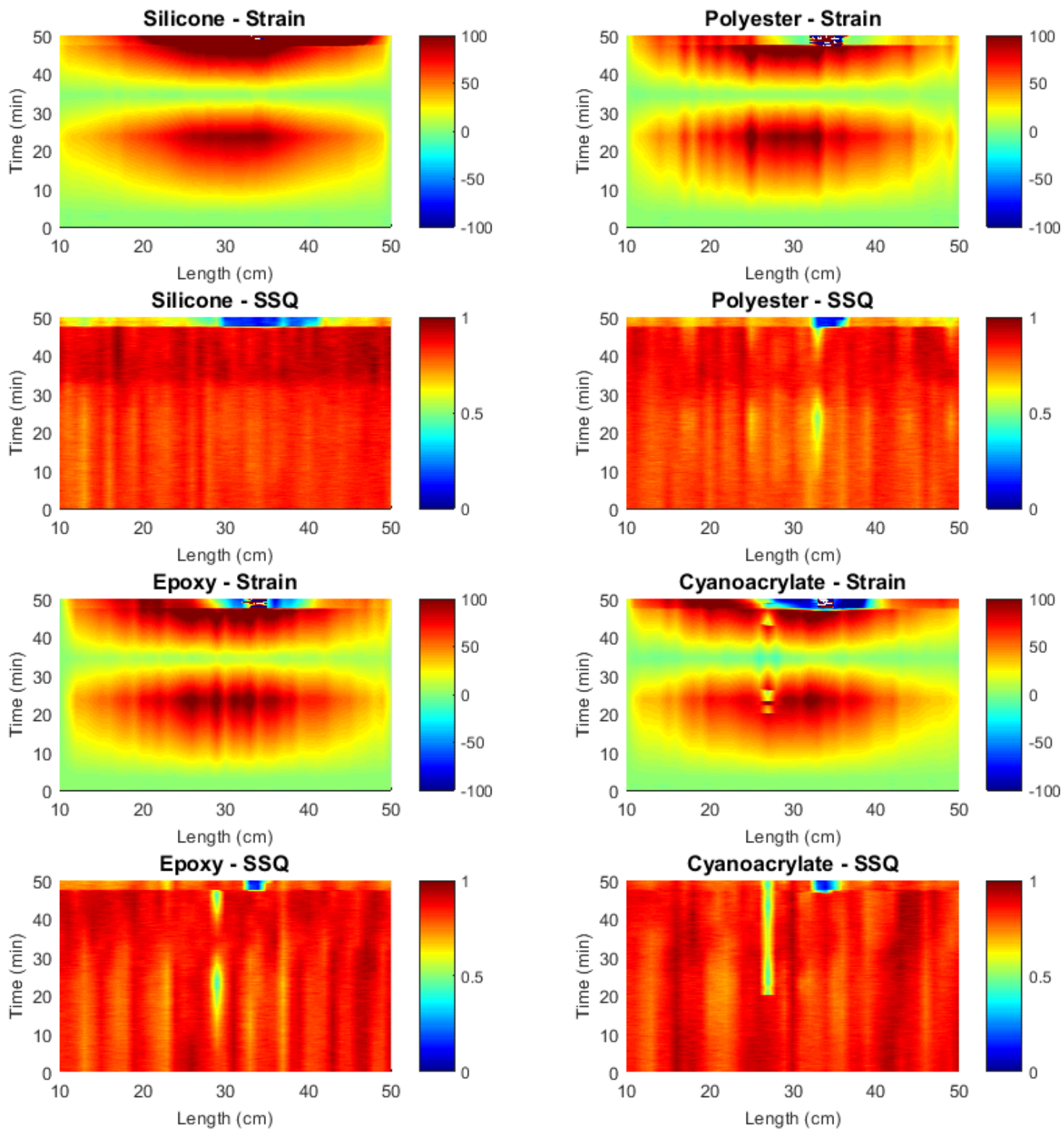

Figure 18. Comparison of SSQ values of each segment with different adhesives when cracking occurs

In the polyester and epoxy bonded segments it is seen how these SSQ values increase again during the unloading sequence of the beam before decreasing again with 
the second loading stage. However, in the cyanoacrylate bonded segment this recovery is not observed. Furthermore, it seems that although above the threshold of 0.15 , in the later segment this drop of the SSQ values has an influence in the strain measurements since at this location the measured strain displays some incoherencies.

An additional take away from this analysis is that in the polyester, epoxy and cyanoacrylate bonded segments, the sudden decrease of the SSQ values in the post-cracking stage, is limited to the immediate whereabouts of the crack location and translates in extremely high strain peaks. On the other hand, in the silicone bonded segment, the area that corresponds to low SSQ values is relatively wider but with strain values within a reasonable range.

In an attempt to better assess the performance of the DOFS measurements after the occurrence of cracking, the authors decided to perform a post-processing routine where the DOFS data points corresponding to a SSQ value below 0.15 were removed. Moreover, after the conduction of this action, there were still some data points, which presented negative values. Naturally, this is not coherent to what is expected in a load test where the entire length of the bottom surface of the beam is in tension. This is due to the proximity of these points to the aforementioned inaccurate values (with SSQ lower than 0.15). In this way, these values are also removed in this routine. Finally, a surface interpolation is conducted replacing the removed data points. This is done along the same lines as the authors have conducted successfully in the past [6].

The results of this routine are depicted in Figure 19 and Figure 20, where the measurements between minute 45 and 50 of the load sequence are displayed. As it is possible to observe from these illustrations, considering the conditions mentioned above, the silicone bonded segment is the one presenting less inaccurate data and consequently having less removed measurements. For the other segments, especially due to the 
removing of negative strain measurements, more data had to be removed. This is then reflected in the interpolated data displayed in Figure 20, especially on the cyanoacrylate bonded segment.
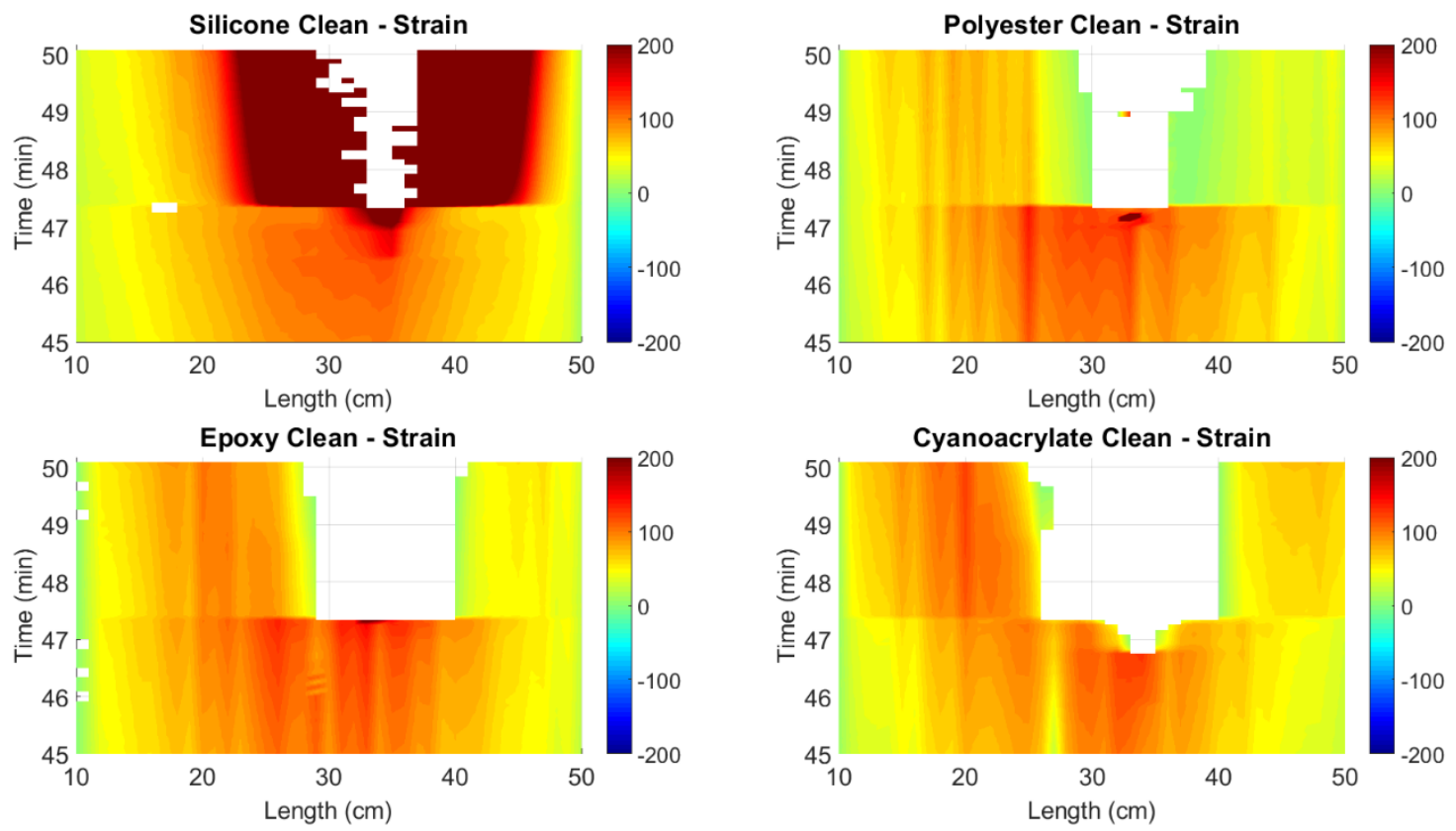

Figure 19. Cleansed DOFS data using SSQ values bellow 0.15 and negative strain
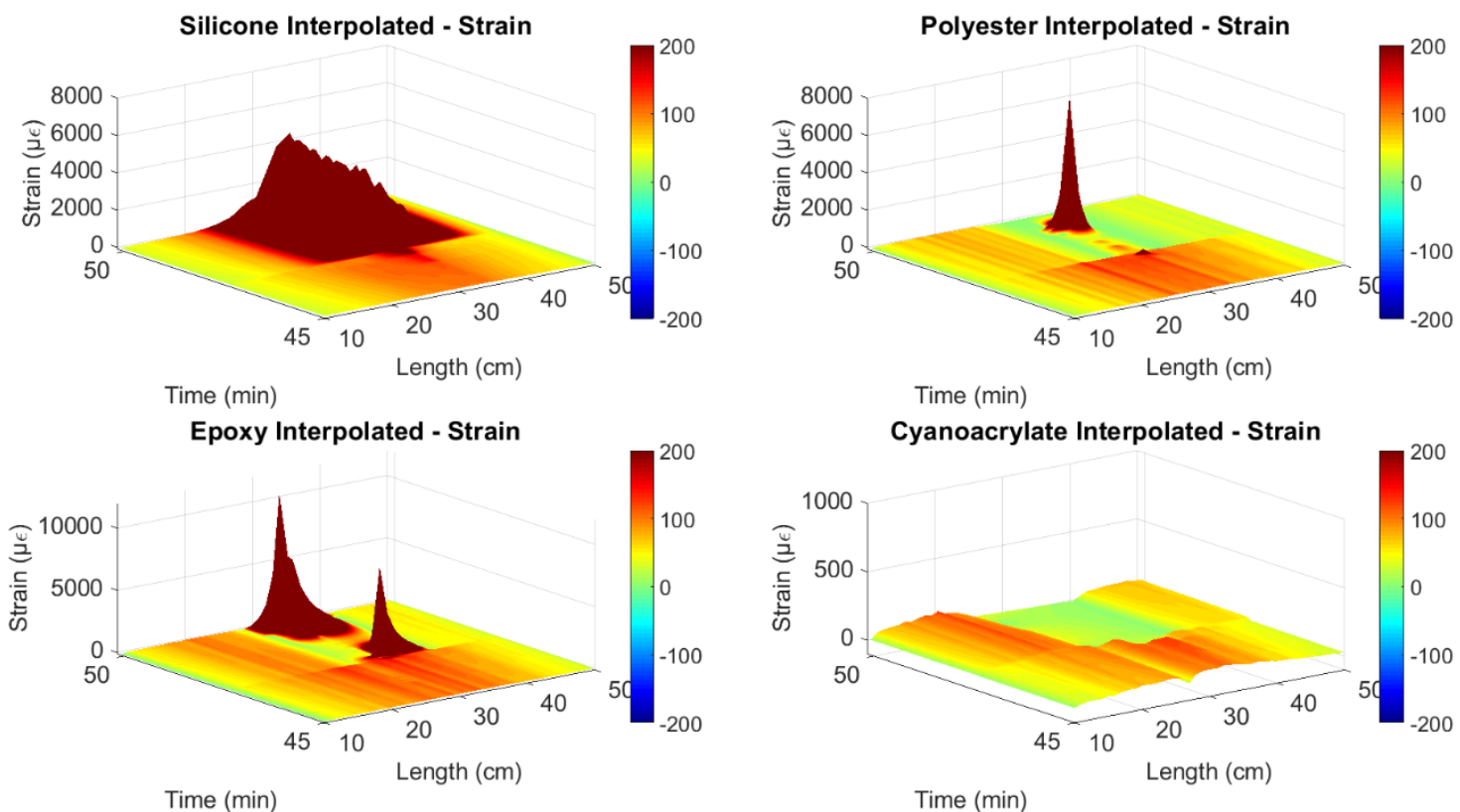

Figure 20. Interpolated strain data

In summary, it is observed a relative smoother performance of the neutral cure silicone during the elastic loading of the concrete beam. All bonded segments presented 
an acceptable agreement with the data collected from the strain gauges where the difference between both set of sensors was around the resolution of the used system of $\pm 2 \mu \varepsilon$.

When cracking appears, all segments are able to detect the formation of the crack. However, this is followed by a sharp decrease of the spectral shift quality (SSQ) at the location of the crack. Moreover, apart from the silicone segment, the measurements become erratic at this location of the beam until the rupture of the fiber. By performing a post-processing routine where the data points corresponding to inaccurate or incoherent values are removed and replaced by interpolating the surrounding accurate data points, it is observed how the silicone segment displays information that is more reliable.

The authors are currently conducting a parallel study with the goal of identifying the possible causes of the drop of SSQ and will present further results in the future.

\section{Conclusions}

In this paper, an experimental campaign to assess the performance of different adhesives for the bonding of polyimide distributed optical fiber sensors in concrete surfaces was presented. Additionally, the influence of the spatial resolution of the DOFS used in the data acquisition was conducted as well.

A three-point load test was carried out on a reinforced concrete beam where a DOFS was bonded to the bottom surface with four segments using different adhesives. Initially, the beam is tested under elastic regime (un-cracked condition) and in second part, the beam is cracked and loaded up to failure.

The results show how the use of a spatial resolution of $1 \mathrm{~mm}$, although theoretically better for an enhanced strain measurement presented an undesired high spatial variability due to the heterogeneity of the aggregates present in the concrete. This 
does not occur with a spatial resolution of $1 \mathrm{~cm}$, which presented a good correlation with the data measured by the strain gauges.

Additionally, after performing a moving average of both the strain data acquired using a $1 \mathrm{~mm}$ and $1 \mathrm{~cm}$ spatial resolution in order to directly compare it with the $3 \mathrm{~cm}$ measured strain, it was seen how the use of $1 \mathrm{~cm}$ resolution provided very similar results. In conclusion, the use of a spatial resolution of $1 \mathrm{~cm}$ is suitable and recommended for this type of applications.

Moving forward to the comparison of the different used bonding adhesives, it was observed how in un-cracked condition, all segments compared fairly well with the data measured by strain gauges along the bottom surface of the concrete. Nevertheless, the silicone bonded segment presented a smoother and more uniform data than the other bonded segments. Therefore, if cracking is not foreseen, all adhesives can be used to obtain accurate and reliable results.

Afterwards, when the tensile capacity of the concrete was exceeded, it was verified how all the segments were able to detect and locate the crack formation. Notwithstanding, the associated SSQ values of the measurements quickly dropped below an acceptable threshold (0.15) shortly after this crack initiation in the whereabouts of the crack location. Therefore, the measurements at this location became erratic in all segments except for the silicone bonded one. Nevertheless, in this later segment the length influenced by the crack formation is relatively wider which compromises the detection on new possible cracks that can have origin in the immediate surroundings of the first detected one.

When removing and replacing the inaccurate and incoherent measurements and replacing them in an interpolation routine, the silicone bonded segment also provide the most realistic results. 
This different behaviour of the silicone bonded segment both for the undamaged and damaged scenarios is related with the relatively low shear modulus presented by this adhesive. As seen, this presents both some advantages as disadvantages depending on the objectives of a possible application of this technology.

In scenarios where the strain variation and quantification of number of cracks for a narrow length of the specimen are not important, the use of this adhesive becomes ideal as it smooths out the obtained readings, although for high strain values in cracked regions the readings may become less accurate. Furthermore, the authors believe that the use of this adhesive would allow for a higher chance of surviving wider crack openings. Notwithstanding, for the case where it is intended to measure large strain gradients and clearly differentiate them in narrower areas, the use of a different adhesive is advised.

Finally, it is important to mention the importance of the viscosity and time of curing of each of the adhesives used. In real and larger structural elements, these parameters are of great importance on the decision of the optimal adhesive for each particular application, which may sway the preference of one adhesive over another.

\section{Acknowledgements}

This project has received funding from the European Union's Horizon 2020 research and innovation programme under the Marie Sklodowska-Curie grant agreement No. 642453.

\section{Conflicts of Interest}

The authors declare no conflict of interest.

\section{References}

[1] P. Ferdinand, "The Evolution of Optical Fiber Sensors Technologies During the 35 Last Years and Their Applications in Structure Health Monitoring," in EWSHM-7th European Workshop on Structural Health Monitoring, 2014. 
[2] A. Barrias, J. Casas, and S. Villalba, “A Review of Distributed Optical Fiber Sensors for Civil Engineering Applications," Sensors, vol. 16, no. 5, p. 748, May 2016 .

[3] S. Villalba and J. R. Casas, "Application of optical fiber distributed sensing to health monitoring of concrete structures," Mech. Syst. Signal Process., vol. 39, no. 1, pp. 441-451, 2012.

[4] G. Rodriguez, J. R. Casas, and S. Villalba, "Cracking assessment in concrete structures by distributed optical fiber," Smart Mater. Struct., vol. 24, no. 3, p. $35005,2015$.

[5] A. Barrias, G. Rodriguez, J. R. Casas, and S. Villalba, "Application of distributed optical fiber sensors for the health monitoring of two real structures in Barcelona," Struct. Infrastruct. Eng., 2018.

[6] A. Barrias, R. J. Casas, and S. Villalba, "Embedded Distributed Optical Fiber Sensors in Reinforced Concrete Structures-A Case Study," Sensors , vol. 18, no. 4. 2018.

[7] M. Davis, N. A. Hoult, and A. Scott, "Distributed strain sensing to determine the impact of corrosion on bond performance in reinforced concrete," Constr. Build. Mater., vol. 114, pp. 481-491, 2016.

[8] R. Regier and N. A. Hoult, "Concrete deterioration detection using distributed sensors," Proc. Inst. Civ. Eng. Build., vol. 168, no. 2, pp. 118-126, 2015.

[9] B. Glišić, D. Posenato, and D. Inaudi, "Integrity monitoring of old steel bridge using fiber optic distributed sensors based on Brillouin scattering," 14th Int. Symp. Smart Struct. Mater. Nondestruct. Eval. Heal. Monit., vol. 6531, no. 2007, p. $65310 \mathrm{P}-65310 \mathrm{P}-8,2007$.

[10] S. T. Kreger, D. K. Gifford, M. E. Froggatt, B. J. Soller, and M. S. Wolfe, "High resolution distributed strain or temperature measurements in single-and multimode fiber using swept-wavelength interferometry," in Optical Fiber Sensors, 2006, p. ThE42. 
[11] LUNA, "Fiber optic sensing," 2014.

[12] Luna Innovations Incorporated, "High-Definition Fiber Optic Strain Sensors." 2016.

[13] D. Inaudi and B. Glisic, "Distributed fiber optic strain and temperature sensing for structural health monitoring," in The Third International Conference on Bridge Maintenance, Safety and Management, IABMAS 2006, pp. 963-964.

[14] F. Ansari and Y. Libo, "Mechanics of bond and interface shear transfer in optical fiber sensors,” J. Eng. Mech., vol. 124, no. 4, pp. 385-394, 1998.

[15] K. T. Wan, C. K. Y. Leung, and N. G. Olson, "Investigation of the strain transfer for surface-attached optical fiber strain sensors," Smart Mater. Struct., vol. 17, no. 3, p. 35037, 2008.

[16] J. M. Henault et al., "Analysis of the strain transfer mechanism between a truly distributed optical fiber sensor and the surrounding medium," in Concrete Repair, Rehabilitation and Retrofitting III: 3rd International Conference on Concrete Repair, Rehabilitation and Retrofitting, ICCRRR-3, 3-5 September 2012, Cape Town, South Africa, 2012, p. 266.

[17] R. Regier, "Application of fibre optics on reinforced concrete structures to develop a structural health monitoring technique," Canadian theses, 2013.

[18] G. Rodríguez, J. R. . Casas, S. Villalba, and A. Barrias, "Monitoring of shear cracking in partially prestressed concrete beams by distributed optical fiber sensors," in Proceedings 8th International Conference on Bridge Maintenance, Safety and Management, IABMAS 2016, 2016.

[19] TML, “TML STRAIN GAUGES 2017.” Tokyo Sokki Kenkyujo Co., Ltd., 2017.

[20] J. R. Pedrazzani, S. M. Klute, D. K. Gifford, A. K. Sang, and M. E. Froggatt, "Embedded and surface mounted fiber optic sensors detect manufacturing defects and accumulated damage as a wind turbine blade is cycled to failure," Luna Innov. Inc, 2012.

[21] Luna Innovations Incorporated, “ODiSI-A Users Guide,” Blacksburg, VA, 2013. 